\title{
An empirical evaluation of four variants of a universal species-area relationship
}

The Maximum Entropy Theory of Ecology (METE) predicts a universal species-area relationship (SAR) that can be fully characterized using only the total abundance $(N)$ and species richness $(S)$ at a single spatial scale. This theory has shown promise for characterizing scale dependence in the SAR. However, there are currently four different approaches to applying METE to predict the SAR and it is unclear which approach should be used due to a lack of empirical comparison. Specifically, METE can be applied recursively or a non-recursively and can use either a theoretical or observed species-abundance distribution (SAD).We compared the four different combinations of approaches using empirical data from 16 datasets containing over 1000 species and 300,000 individual trees and herbs. In general, METE accurately downscaled the SAR $\left(R^{2}>0.94\right)$, but the recursive approach consistently under-predicted richness, and METE's accuracy did not depend strongly on using the observed or predicted SAD. This suggests that the best approach to scaling diversity using METE is to use a combination of non-recursive scaling and the theoretical abundance distribution, which allows predictions to be made across a broad range of spatial scales with only knowledge of the species richness and total abundance at a single scale. 
1 Daniel J. McGlinn $^{1 *}$, Xiao Xiao ${ }^{1}$, and Ethan P. White ${ }^{1}$

2 1. Department of Biology and the Ecology Center, Utah State University, Logan, UT, 84341, 3 USA.

4

$5 *$ corresponding author 
Introduction:

The species-area relationship (SAR) is a fundamental ecological pattern that characterizes

8 the change in species richness as a function of spatial scale. The SAR plays a central role in

9 predicting the diversity of unsampled areas (Palmer 1990), reserve design (Whittaker et al. 2005),

10 and estimating extinction rates due to habitat loss (Brooks et al. 2004). Applications involving the

11 SAR depend strongly on the form of the relationship (Guilhaumon et al. 2008) which is known to

12 change with spatial scale (Palmer and White 1994, McGlinn and Hurlbert 2012). Despite the

13 scale-dependence of the SAR, a simple non-scale dependent model (the power-law) is still the

14 most commonly used model for the SAR (Tjørve 2003).

15 The Maximum Entropy Theory of Ecology (METE) is a unified theory that shows

16 promise for characterizing a variety of macroecological patterns including the species-abundance

17 distribution, a suite of relationship between body-size and abundance, and a number of spatial

18 patterns including the species-area relationship (Harte et al. 2008, 2009, Harte 2011). METE

19 adopts the inferential machinery of Maximum Entropy (MaxEnt; Jaynes 2003) to solve for the

20 most likely state of an ecological community (Haegeman and Loreau 2008, 2009) using only

21 information on the total number of species, the total number of individuals, the total metabolic

22 rate of all the individuals, and the area of the community.

23 METE predicts that all SARs follow a universal relationship between the exponent of a

24 power-law characterizing the SAR at a particular scale and the ratio of richness and community

25 abundance. The exponent of the SAR is scale dependent, decreasing with increasing spatial scale.

26 Empirical evaluation of the theory suggests that METE is a promising model for the SAR (Harte

27 et al. 2008, 2009); however, there are currently four different approaches to applying METE to

28 predict the SAR and it is unclear which approach should be used due to a lack of empirical

29 comparison. 
There are two distinct versions of METE, recursive (where richness at different scales is

31 obtained by consecutively halving or doubling of area; Harte et al. 2009) and non-recursive

32 (where richness at different scales is obtained directly; Harte et al. 2008), which predict

33 somewhat different SARs. It is not clear a priori which of these versions of METE should be

34 more accurate, and it has been suggested that the best approach should be chosen using empirical

35 comparisons (Harte 2011). In addition, the METE-SAR is derived using the species-abundance

36 distribution (SAD), which can be predicted from $N$ and $S$ or by using the empirical distribution.

37 The most general use of METE for predicting diversity across scales relies on the use of the

38 theoretical abundance distribution, but there have been no comparisons of METE-SAR

39 predictions using theoretical and empirical SADs.

40 To understand which approach to METE is best for characterizing diversity across scales we

41 conducted a thorough empirical comparison of the four different variants of the METE-SAR

42 prediction: 1) recursive with predicted $\mathrm{SAD}, 2$ ) recursive with observed $\mathrm{SAD}, 3$ ) non-recursive

43 with predicted SAD, and 4) non-recursive with observed SAD. Using 16 spatially explicit plant

44 datasets we compared the form and accuracy of the predicted SAR across the four variations of

45 METE at a wide-range of spatial scales and across a diverse set of plant communities with over

461000 species and 300,000 individual trees and herbs.

\section{Methods:}

48 Downscaling Richness

49 The METE approach to predicting the SAR is a two-step application of the maximum

50 entropy formalism (MaxEnt): 1) MaxEnt is first used to predict the SAD which represents the

51 probability that a species has abundance $n_{0}$ in a community of area $A_{0}$ with $S_{0}$ species and $N_{0}$

52 individuals, $\varphi\left(n_{0} \mid N_{0}, S_{0}, A_{0}\right)$, and 2) MaxEnt is then used to predict the intra-specific, spatial-

53 abundance distribution which represents the probability that $n$ out of $n_{0}$ individuals of a species

54 are located in a random quadrat of area $A$ drawn from a total area $A_{0}, \Pi\left(n \mid A, n_{0}, A_{0}\right)$. The $\Pi$ 
55 distribution is spatially implicit and does not contain information on the spatial correlation

56 between cells. If the observed species abundance distribution is used instead of the METE

57 distribution, then only the $\Pi$ distribution is solved for using MaxEnt.

There are no adjustable parameters in METE, and the solutions to $\Pi$ and $\varphi$ only depend

59 on the empirical constraints and possible system configurations (Haegeman and Loreau 2008, 2009, Haegeman and Etienne 2010). If the observed SAD is not used then constraints on the average number of individuals per species $\left(N_{0} / S_{0}\right)$ and on the upper bound of the number of individuals $N_{0}$ can be used to yield a truncated log-series abundance distribution (Fig. 1, Harte et al. 2008, Harte 2011). To predict $\Pi$, METE places constraints on the average number of individuals per unit area $\left(n_{0} A / A_{0}\right)$ and on the upper bound of the total abundance of a species $n_{0}$. Although METE requires total metabolic rate to derive its predictions, this variable can be ignored when solving for the METE SAD or SAR (Harte et al. 2008, 2009, Harte 2011). recursive approach (Fig. 1a,b) in which the constraints at $A_{0}$ are used to solve for $\Pi$ at $A_{0} / 2$, which provides new constraints (i.e., predicted $S_{\mathrm{Ao} / 2}$ and $N_{\mathrm{Ao} / 2}$ ) for solving $\Pi$ at $A_{0} / 4$ and so on until richness is computed at every bisection of the total area $\mathrm{A}_{0} / 2^{i}$ where $i$ is a positive integer (Harte et al. 2009, Harte 2011, p159). The recursive approach continually updates its prior information as it downscales richness. Alternatively we can use a non-recursive approach (Fig. 1c,d) in which

73 we solve for $\Pi$ at any area based only on the constraints at $A_{0}$ (Harte et al. 2008, Harte 2011,

74 p243). The recursive approach may be more accurate because it continually upgrades its prior

75 information or less accurate due to error propagation thus only empirical comparisons can

76 determine which approach is best used for prediction (Harte 2011, p160). Harte (2011) provides the derivations for the $\Pi$ and $\Phi$ distributions, so here we will only

78 highlight the most relevant equations for differentiating the four METE variants. The MaxEnt solution to maximizing entropy for $\Pi$ is: 
80

$$
\Pi\left(n \vee A, n_{0}, A_{0}\right)=\frac{1}{Z_{\Pi}} e^{-\lambda_{\Pi} n}
$$

81

82 where $\lambda_{\Pi}$ is the unknown Lagrange multiplier and $Z_{\Pi}$ is the partition function (Harte 2011, Eq.

83 7.48). The partition function ensures normalization and it is defined as:

84

$$
Z_{\Pi}=\sum_{n=0}^{n_{0}} e^{-\lambda_{\Pi} n}=\frac{1-e^{-\lambda_{\Pi}\left(n_{0}+1\right)}}{1-e^{-\lambda_{\Pi}}}
$$

86 The Lagrange multiplier can be solved for by defining $\Pi$ in terms of its constraints which yields:

$$
\bar{n}=\frac{n_{0} A}{A_{0}}=\frac{\sum_{n=0}^{n_{0}} n x^{n}}{\sum_{n=0}^{n_{0}} x^{n}}\left(\frac{x}{1-x}-\frac{\left(n_{0}+1\right) \cdot x^{n_{0}+1}}{1-x^{n_{0}+1}}\right)
$$

89 where $x=e^{-\lambda_{\Pi}}$ to simplify notation. Although the METE prediction for $\Pi$ can be solved

90 numerically for any area, it is only known analytically for a special case in which the area $A$ is

91 half the total area $A_{0}$ (Harte 2011, Eq. 7.51):

92

$$
\Pi\left(n \vee \frac{A_{0}}{2}, n_{0}, A_{0}\right)=\frac{1}{1+n_{0}}
$$

93 Eq. 4 shows that METE predicts that all possible arrangements of $n_{0}$ individuals are equally likely

94 across two equal area quadrats. The flat distribution characterized by Eq. 4 is identical to the

95 prediction offered by the Hypothesis of Equal Allocation Probability (HEAP) model and

96 therefore the recursive application of Eq. 4 to downscale $\Pi$ generates the same set of $\Pi$

97 distributions as the HEAP model (Harte et al. 2005, Harte 2007, 2011): 


$$
\Pi\left(n \vee \frac{A_{0}}{2^{i}}, n_{0}, A_{0}\right)=\sum_{q=n}^{n_{0}} \frac{\Pi\left(q \vee \frac{A_{0}}{2^{i-1}}, n_{0}, A_{0}\right)}{(q+1)}, i=1,2,3, \ldots
$$

100 Thus for a given bisection of the total area (i.e., $A=A_{0} / 2^{i}$ ) we can either use the recursive

101 approach (Eq. 5) or the non-recursive approach (Eq. 1) to compute the $\Pi$ distribution.

Expected richness is simply the sum of the individual probabilities of species occupancy.

103 Table 1 gives the expressions for expected richness at $A_{0} / 2$ given the four possible combinations

104 of the choice of the downscaling approach and the choice of SAD to use. The equations in Table

1051 will also hold for finer spatial scales except for the recursive, theoretical SAD approach which 106 requires downscaling the SAD as well (Harte 2011, Eq. 7.63).

\section{Empirical Comparison}

108 Testing METE's predictions requires spatially explicit, contiguous data from a single

109 trophic level. We carried out an extensive search for open data that met these requirements. This

110 search resulted in a database of 16 communities (Table 2; see Supplemental Information: Table

111 S1 for additional details). All of the datasets are terrestrial, woody plant communities with the

112 exception of the serpentine grassland which is herbaceous. In the woody plant surveys, the

113 minimum diameter at breast height (i.e., $1.4 \mathrm{~m}$ from the ground) that a tree must be to be included

114 in the census was $10 \mathrm{~mm}$ with the exception of the Cross Timbers and Oosting sites where the

115 minimum diameter was 25 and $20 \mathrm{~mm}$ respectively. Where datasets contained time-series

116 information we selected a single census year from each dataset to analyze. Harte (2011)

117 suggested that MaxEnt models will perform best when a single process such as the presence of a

118 past disturbance is not dominating the system and rather a multitude of different interacting

119 processes are operating. With this in mind, we attempted to choose the survey years that were the

120 longest amount of time from known stand-scale disturbances (e.g., hurricane events). 
122 2003). Recursive METE only makes predictions for bisected areas so we restricted our datasets to

123 areas that were square or rectangular with the dimensional ratio of 2:1. Due to the irregular shape

124 of the Sherman and Cocoli sites we defined two separate $200 \times 100 \mathrm{~m}$ subplots within each site

125 (Supplemental Information: Fig. S1). We then calculated the results for each of the two subplots

126 and reported the average.

127 To assess the accuracy of METE's predictions for the SAD and the four downscaling 128 algorithms of the SAR, we computed the coefficient of determination about the one-to-one line:

$$
\begin{aligned}
& o b s_{i}-\operatorname{pred}_{i} \dot{b}^{2} \\
& \qquad b s_{i}-\overline{o b s}_{i} \dot{b}^{2} \text { where } o b s_{i} \text { and } \text { pred }_{i} \text { are the } i \text { th log-transformed observed and METE-predicted } \\
& \quad \stackrel{\dot{i}}{R^{2}}=1-\sum \dot{i}
\end{aligned}
$$

130 values (abundance for the SAD, richness for the SAR) respectively. Log transformed richness

131 was used to minimize the influence of the few very large richness values and because relative

132 deviations are of greater interest in evaluating SARs than absolute differences. We used the

133 python package METE (White et al. 2013), as well as, a suite of project specific R and python

134 scripts for our analysis. All R and Python code used to generate these analyses is archived in the

135 supplemental materials and also available on GitHub (http://github.com/weecology/mete-spatial).

\section{Results}

The four versions of METE all produced reasonable estimates of downscaled richness

138 (Fig. 1). The $R^{2}$ values ranged from 0.944 for the recursive, observed-SAD model up to 0.997 for

139 the non-recursive, observed-SAD (Fig. 2). Despite the high coefficient of determination, the

140 recursive approach deviated systematically from the empirical data by underpredicting richness 
141 (Fig. 2,3). This deviation became larger at finer scales (Fig. 2). In contrast, the non-recursive

142 approach showed no systemic deviations. The SAD was well characterized by the METE

143 predictions $\left(R^{2}=0.95\right)$; however, METE did on average predict slightly more uneven

144 communities (i.e., predicted abundance was too low for rare species and too high for abundant

145 species, Supplemental Information: Fig. S2). Overall, the inclusion of the observed SAD did not

146 strongly improve the prediction of the SAR. For the non-recursive approach including the

147 observed SAD improved the overall $R^{2}$ from 0.984 to 0.997 (Fig. 3c,d), but the accuracy of the

148 recursive model actually decreased with the inclusion of the observed SAD $\left(R^{2}\right.$ from 0.976 to

149 0.944, Fig. 3a, b).

150 Results were broadly consistent across datasets, with the exception of the serpentine

151 grassland and Cross Timbers oak woodland. The serpentine community displayed a steeper non-

152 saturating SAR in contrast to the other datasets, and was the only dataset where the recursive

153 downscaling approach was more accurate (Fig. 2o). The oak community displayed a sigmoidal

154 SAR, and in contrast to the other study sites the inclusion of the observed SAD for the oak

155 community resulted in a large improvement in the predicted SAR (Fig. 2p).

\section{Discussion}

All four variations of METE performed well at predicting species richness across scales

158 (all $R^{2}>0.94$ ); however, some versions performed consistently better than others. The non-

159 recursive approach outperformed the recursive version of METE in all but one dataset (the

160 serpentine grassland). The recursive approach also showed small, but consistent, under-

161 predictions for species richness. This means that the recursive approach predicted stronger intra-

162 specific spatial aggregation than observed in the data. This finding is consistent with Harte's

163 (2011) comparisons of the species-level spatial abundance distribution in which the recursive

164 approach predicted greater aggregation than the non-recursive approach. Given that the recursive 165 approach provides a poorer fit to empirical data and can only be applied at particular scales (i.e., 
$\left.166 \mathrm{~A}_{0} / 2, \mathrm{~A}_{0} / 4, \ldots\right)$, we recommend the use of the non-recursive approach for downscaling the SAR.

167 However, the recursive approach is currently the only means of providing a METE-based

168 prediction for the distance decay relationship via the hypothesis of equal allocation probabilities

169 approach in Harte (2007), and the universal relationship between $S / N$ and the slope of the SAR is

170 currently only known for the recursive approach (Harte et al. 2009).

171 The SAR predictions were generally robust to using the predicted rather than observed

172 SAD. Including the observed SAD increases the amount of information used to constrain the

173 predictions, but it did not substantially increase the overall accuracy of the SAR predictions. This

174 was primarily because the empirical SAD was well characterized by the METE-SAD, consistent

175 with several other studies (Harte et al. 2008, Harte 2011, White et al. 2012). Models in general,

176 and MaxEnt models in particular, typically match empirical data better as increasing numbers of

177 parameters or constraints are included in the analysis (Haegeman and Loreau 2008, Roxburgh

178 and Mokany 2010, Harte 2011). Therefore the naïve expectation for using the observed SAD is

179 that the accuracy of the prediction should increase. However, this was generally only true for the

180 non-recursive approach. This occurred because rarity and intraspecific aggregation interact in

181 subtle ways to determine the shape of the SAR (He and Legendre 2002, McGlinn and Palmer

182 2009), and simply fixing one of these pieces of information does not guarantee improved

183 predictive power. While using the observed SAD does improve the $R^{2}$ for the non-recursive form

184 of METE, it only does so by $\sim 1 \%$. Therefore $N$ and $S$ are generally sufficient to accurately

185 downscale richness using METE across a wide range of habitat types. This is important because

186 it should be possible to model geographic patterns of richness and abundance at a single scale to

187 predict the SAD (White et al. 2012) and then use those modeled values to predict richness across 188 scales.

189 Although METE yields accurate predictions for the SAR, its current form has limitations 190 with respect to its extent of applicability and its ability to tie in more broadly with species-time 
191 and species-time-area relationships (Rosenzweig 1995, White et al. 2010). Specifically, METE

192 predictions are thought to be most relevant for single trophic level datasets that are spatially

193 contiguous and relatively environmentally homogenous (Harte 2011), thus constraining the

194 applicability of METE. At the large spatial scales that are often of interest in conservation

195 planning it is likely that a standard application of METE will fail once species ranges do not

196 occupy all of $A_{0}$. These are also the scales at which the third phase of the triphasic SAR is

197 expected to occur (Allen and White 2003, Storch et al. 2012), and METE does not predict this

198 accelerating phase. However, McGill (2010) suggested that METE's local predictions could be

199 connected with a broad-scale theory to predict a triphasic SAR. Additionally, METE does not

200 currently make predictions through time; however, Harte (2011) suggests using Maximum

201 Entropy Production (Dewar 2005). It should be possible to extend METE to predict the species-

202 time-area relationship (White et al. 2010) because this pattern, like the SAR, can be modeled in

203 terms of the number of unique individuals sampled per unit area and time (McGlinn and Palmer

204 2009).

Recently there have been two critiques of the METE spatial predictions. The universality

206 of the relationship between the slope of the recursive METE-SAR and the ratio of N/S was

207 questioned on the basis that the predicted METE-SAR for subsets of a community cannot be

208 added to yield the community based prediction (Šizling et al. 2011, 2013). However, Harte et al.

209 (2013) argue that it is not a flaw of METE or a strong argument against universality. Additionally,

210 Haegeman and Etienne (2010) argued that a multivariate, spatially implicit analog of the

211 univariate $\Pi$ distribution that is derived using the non-recursive METE approach makes different

212 predictions at different spatial scales (i.e., it is not scale consistent); however, they recognize that

213 a spatially-explicit, scale-consistent version of this distribution may still exist. This critique does

214 not apply to the recursive approach (McGlinn et al. unpublished data), but it may apply in other

215 contexts such as the scaling of the SAD. The lack of scale-consistency in some of METE's 
216 predictions suggests that the choice of the anchor scale $\left(A_{0}\right)$ may influence the theory's

217 predictions; however, our results which spanned a range of anchor scales (0.0064 to $50 \mathrm{ha}) \mathrm{did}$

218 not appear to change systematically with scale. Furthermore, White et al. (2012) demonstrated

219 that the METE-SAD accurately characterized empirical SADs across studies with a wide range of

220 anchor scales. Although METE may not provide a universal model of spatial structure in

221 ecological systems and some of its predictions will depend on the anchor scale, our results as well

222 as others suggest that METE can be used as a practical tool for inferring patterns of diversity and

223 abundance from relatively little information.

224 We examined the down-scaling of richness; however, many conservation applications are

225 interested in up-scaling richness or predicting diversity at a coarse unsampled scale using

226 information at a fine scale. Harte et al. (2009) demonstrated that recursive-METE accurately up-

227 scaled tropical tree richness. Currently a formal examination of upscaling using the non-recursive 228 approach is lacking. Thus, future investigations should examine the ability of different variants of

229 METE to upscale richness across a range of spatial scales and ecological systems.

230 METE represents a useful practical tool for accurately predicting species richness across

231 spatial scales. Among METE's four different approaches to predict SAR, our analysis

232 demonstrates that the non-recursive approach outperforms the recursive approach, and that using

233 the observed rather than predicted SAD does not substantially improve accuracy. Therefore the

234 METE prediction derived using the non-recursive approach and the predicted SAD will likely be

235 the most useful for future applications involving the SAR.

\section{Acknowledgements}

237 Our manuscript was improved by helpful comments from V. Bahn, J. Harte, J. Kitzes, and

238 one anonymous reviewer. We thank R.K. Peet for providing the oak-hickory and old field pine

239 forest data, J. Green for providing the serpentine grassland data, and J. Arévalo for providing the 240 oak woodland data. 


\section{Literature Cited}

242 Allen, A. P., and E. P. White. 2003. Effects of range size on species-area relationships.

243 Evolutionary Ecology Research 5:493-499.

244 Brooks, T., G. A. B. da Fonseca, and A. S. L. Rodrigues. 2004. Species, data, and conservation $245 \quad$ planning. Conservation Biology 18:1682-1688.

246 Dewar, R. C. 2005. Maximum entropy production and the fluctuation theorem. Journal of Physics A: Mathematical and General 38:L371.

248 Guilhaumon, F., O. Gimenez, K. J. Gaston, and D. Mouillot. 2008. Taxonomic and regional uncertainty in species-area relationships and the identification of richness hotspots. Proceedings of the National Academy of Sciences 105:15458-15463.

Haegeman, B., and R. S. Etienne. 2010. Entropy maximization and the spatial distribution of species. The American Naturalist 175:E74-E90.

Haegeman, B., and M. Loreau. 2008. Limitations of entropy maximization in ecology. Oikos 117:1700-1710.

Haegeman, B., and M. Loreau. 2009. Trivial and non-trivial applications of entropy maximization in ecology: a reply to Shipley. Oikos 118:1270-1278.

Harte, J. 2007. Toward a mechanistic basis for a unified theory of spatial structure in ecological communities at multiple spatial scales. Pages 101-126 in D. Storch, P. A. Marquet, and J. H. Brown, editors. Scaling Biodiversity. . Cambridge University Press, Cambridge. Energetics. . Oxford University Press.

262 Harte, J., E. Conlisk, A. Ostling, J. L. Green, and A. B. Smith. 2005. A theory of spatial structure in ecological communities at multiple spatial scales. Ecological Monographs 75:179-197.

264 Harte, J., J. Kitzes, Newman, and A. J. Rominger. 2013. Taxon Categories and the Universal Species-Area Relationship: A Comment on Šizling et al., "Between Geometry and 
Biology: The Problem of Universality of the Species-Area Relationship.” The American Naturalist 181:282-287.

268 Harte, J., A. B. Smith, and D. Storch. 2009. Biodiversity scales from plots to biomes with a

269 universal species-area curve. Ecology Letters 12:789-797.

270 Harte, J., T. Zillio, E. Conlisk, and A. B. Smith. 2008. Maximum entropy and the state-variable 271 approach to macroecology. Ecology 89:2700-2711.

272 He, F. L., and P. Legendre. 2002. Species diversity patterns derived from species-area models. $273 \quad$ Ecology 83:1185-1198.

274 Jaynes, E. 2003. Probability: the logic of science. . Cambridge University Press, Cambridge. 275 McGill, B. J. 2010. Towards a unification of unified theories of biodiversity. Ecology Letters $276 \quad 13: 627-642$.

277 McGlinn, D. J., and A. H. Hurlbert. 2012. Scale dependence in species turnover reflects variance 278 in species occupancy. Ecology 93:294-302.

279 McGlinn, D. J., and M. W. Palmer. 2009. Modeling the sampling effect in the species-time-area 280 relationship. Ecology 90:836-846.

281 Palmer, M. W. 1990. The estimation of species richness by extrapolation. Ecology 71:1195-1198.

282 Palmer, M. W., and P. S. White. 1994. Scale dependence and the species-area relationship. 283 American Naturalist 144:717-740.

284 Rosenzweig, M. L. 1995. Species Diversity in Space and Time. . Cambridge University Press, 285 Cambridge, UK.

286 Roxburgh, S. H., and K. Mokany. 2010. On testing predictions of species relative abundance 287 from maximum entropy optimisation. Oikos 119:583-590.

288 Scheiner, S. M. 2003. Six types of species-area curves. Global Ecology \& Biogeography 12:441289 447. 
Šizling, A. L., Kunin, and D. Storch. 2013. Taxon-and-Area Invariances, Maximum Entropy, and the Species-Area Relationship: A Reply to Harte et al. The American Naturalist 181:288290.

293 Šizling, A. L., W. E. Kunin, E. Šizlingová, J. Reif, and D. Storch. 2011. Between Geometry and 294 Biology: The Problem of Universality of the Species-Area Relationship. The American Naturalist 178:602-611.

Storch, D., P. Keil, and W. Jetz. 2012. Universal species-area and endemics-area relationships at continental scales. Nature 488:78-81.

298 Tjørve, E. 2003. Shapes and functions of species-area curves: a review of possible models.

299 Journal of Biogeography 30:827-835.

300 White, E. P., S. K. M. Ernest, P. B. Adler, A. H. Hurlbert, and S. K. Lyons. 2010. Integrating

301 spatial and temporal approaches to understanding species richness. Philosophical Transactions of the Royal Society B: Biological Sciences 365:3633-3643.

White, E. P., D. J. McGlinn, X. Xiao, S. Supp, and K. Thibault. 2013. METE - Software for Analyzing Harte et al.'s Maximum Entropy Theory of Ecology. URL: http://dx.doi.org/10.6084/m9.figshare.815905.

White, E. P., K. M. Thibault, and X. Xiao. 2012. Characterizing species abundance distributions across taxa and ecosystems using a simple maximum entropy model. Ecology 93:1772-

309 Whittaker, R. J., M. B. Araujo, J. Paul, R. J. Ladle, J. E. M. Watson, and K. J. Willis. 2005.

310 Conservation Biogeography: assessment and prospect. Diversity and Distributions 11:331123. 


\section{Table 1 (on next page)}

The four variants of METE formulated for expected species richness

The four variants of METE formulated for expected species richness at $A 0 / 2$, given either the recursive or non-recursive method of downscaling and either the theoretical or observed SAD. These equations also hold for finer spatial scales except for the recursive, METE-SAD approach which requires downscaling the SAD as well (see Harte 2011, Eq. 7.63). n0 is the vector of empirical abundances and $\mathrm{n} 0, \mathrm{j}$ is the abundance of the jth species at the community scale $(A 0)$. 


\section{Method of Downscaling}

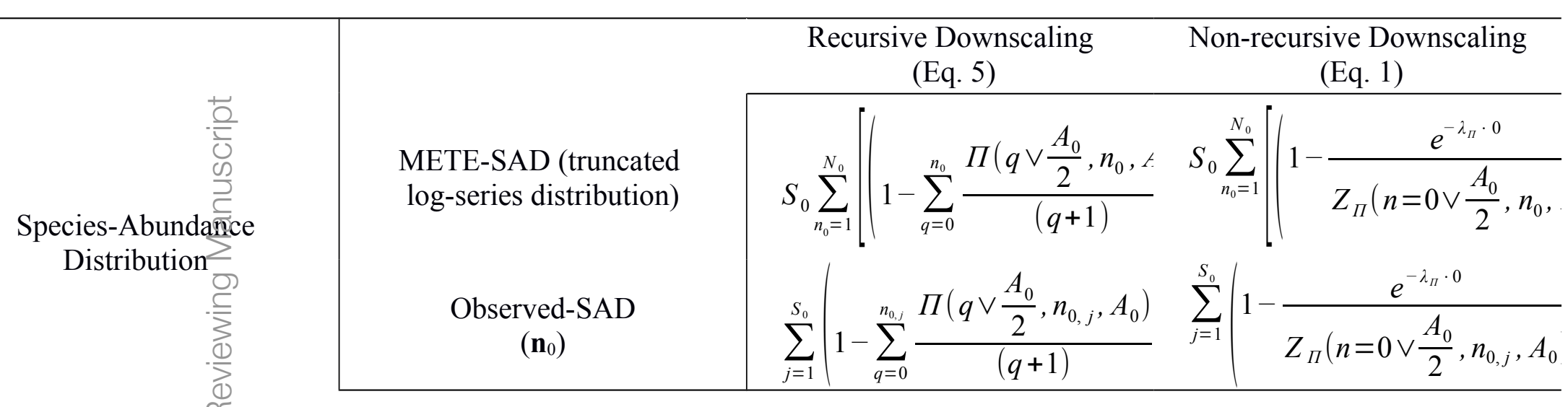




\section{Table 2 (on next page)}

Summary of the datasets

Summary of the habitat type and state variables of the vegetation datasets analyzed. The state variables are total area $\left(A_{0}\right)$, total abundance $\left(N_{0}\right)$ and total number of species $\left(S_{0}\right)$. 


\begin{tabular}{lccccc}
\hline Site names & Habitat type & Ref. & $\boldsymbol{A}_{\mathbf{0}}(\mathbf{h a})$ & $\boldsymbol{N}_{\mathbf{0}}$ & $\boldsymbol{S}_{\mathbf{0}}$ \\
\hline BCI & tropical forest & 1,2 & 50 & 205096 & 301 \\
Sherman & tropical forest & 1 & 2 & 7622.5 & 174.5 \\
Cocoli & tropical forest & 1 & 2 & 4326 & 138.5 \\
Luquillo & tropical forest & 3 & 12.5 & 32320 & 124 \\
Bryan & oak-hickory forest & $4-6$ & 1.7113 & 3394 & 48 \\
Big Oak & oak-hickory forest & $4-6$ & 2 & 5469 & 40 \\
Oosting & oak-hickory forest & 7 & 6.5536 & 8892 & 39 \\
Rocky & oak-hickory forest & $4-6$ & 1.44 & 3383 & 37 \\
Bormann & oak-hickory forest & $4-6$ & 1.96 & 3879 & 30 \\
Wood Bridge & oak-hickory forest & $4-6$ & 0.5041 & 758 & 19 \\
Bald Mtn. & oak-hickory forest & $4-6$ & 0.5 & 669 & 17 \\
Landsend & old field, pine forest & $4-6$ & 0.845 & 2139 & 41 \\
Graveyard & old field, pine forest & $4-6$ & 1 & 2584 & 36 \\
UCSC & mixed evergreen forest & 8 & 4.5 & 5885 & 31 \\
Serpentine & serpentine grassland & 9 & 0.0064 & 37182 & 24 \\
Cross Timbers & oak woodland & 10 & 4 & 7625 & 7 \\
\hline Ranges & & & $0.0064-50$ & $669-205096$ & $7-301$ \\
\hline
\end{tabular}




\section{Figure 1}

An illustration of the process for downscaling species richness

An illustration of the process for downscaling species richness from $A_{0}$ to $A_{0} / 4$ across the four variants of METE. The recursive approach uses either (a) the theoretical SAD (inset curve) or (b) the observed SAD (inset points) to predict richness at $A_{0} / 2$ and then the process is repeated to generate a prediction at $A_{0} / 4$. In contrast, the non-recursive approach uses either (c) the theoretical SAD or (d) the observed SAD to predict richness at $A_{0} / 4$ directly. $S_{0}$ is the total number of species, $N_{0}$ is the total number of individuals, and $\mathbf{n}_{0}$ is the vector of species abundances at the community scale $\left(A_{0}\right)$ abundance.

(a)
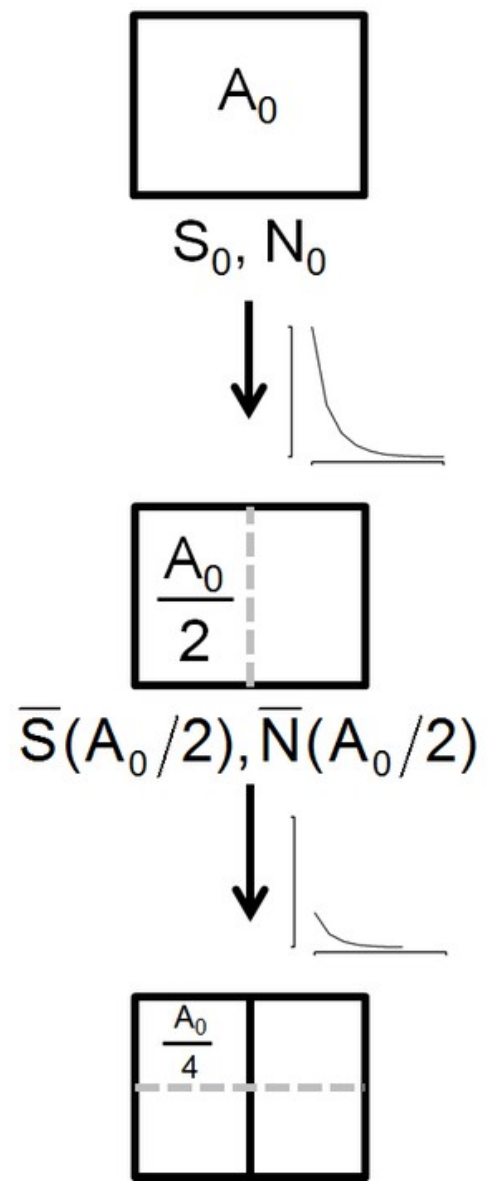

$\bar{S}\left(A_{0} / 2\right), \bar{N}\left(A_{0} / 2\right)$
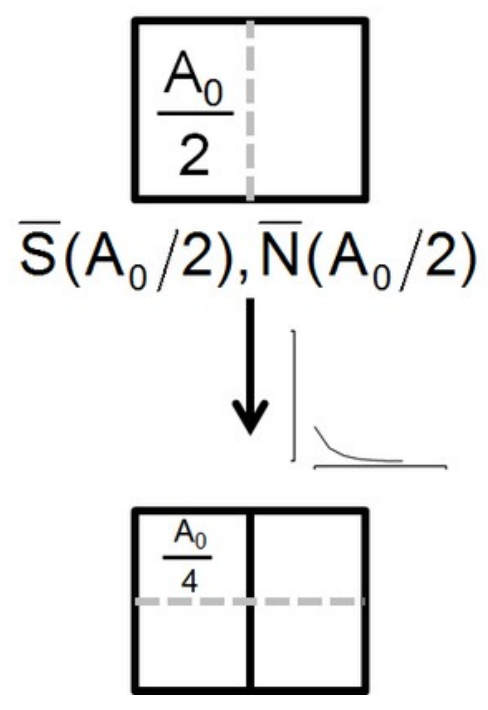

(b)

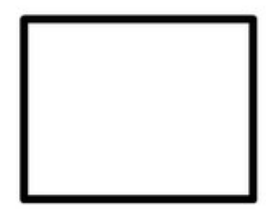

$\mathbf{n}_{0}$
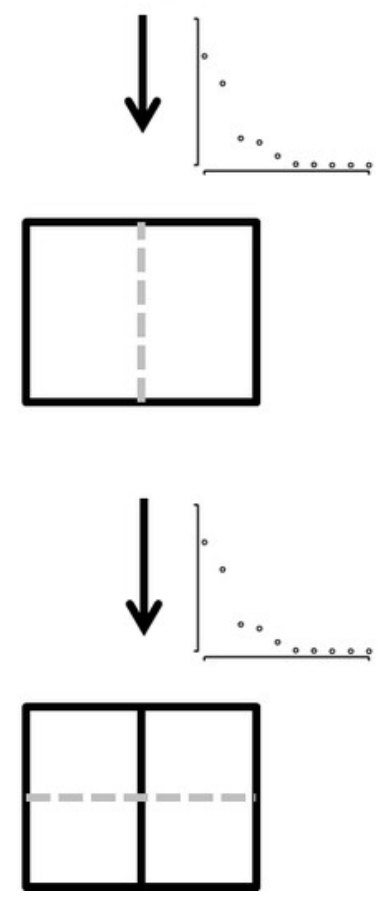

(c)

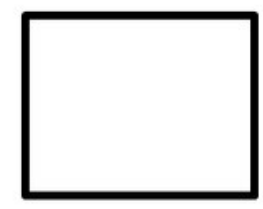

$\mathrm{S}_{0}, \mathrm{~N}_{0}$
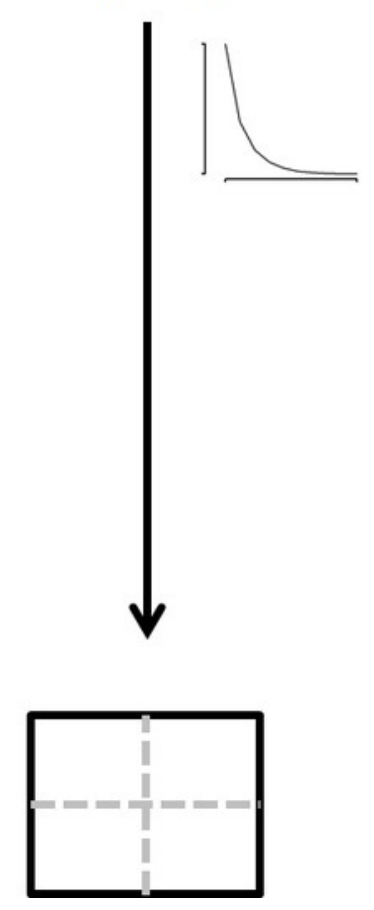

(d)

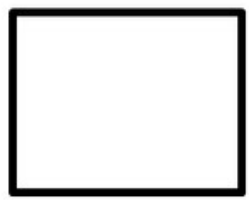

$\mathbf{n}_{0}$

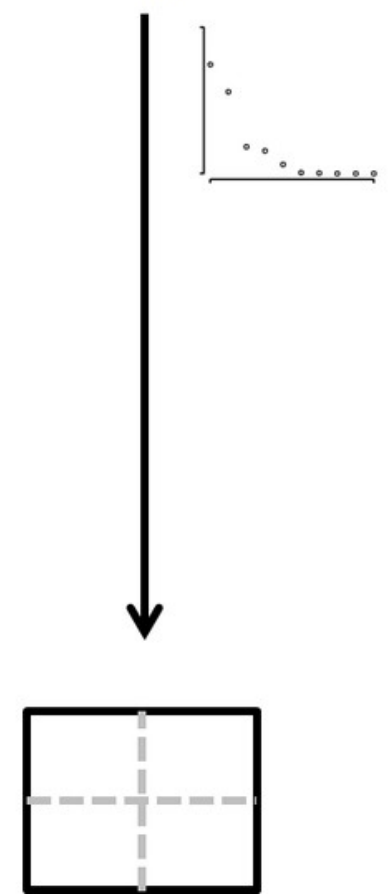




\section{Figure 2}

\section{Empirical species-area relationships}

Empirical species-area relationships and the four versions of the METE model across the 16 sites. The habitat type of each site is given above each panel. The empirical averages are the open circles, the recursive approach is the red lines, the non-recursive approach is the blue lines, the curves using the observed SAD are dashed and those using the METE-SAD are solid. 

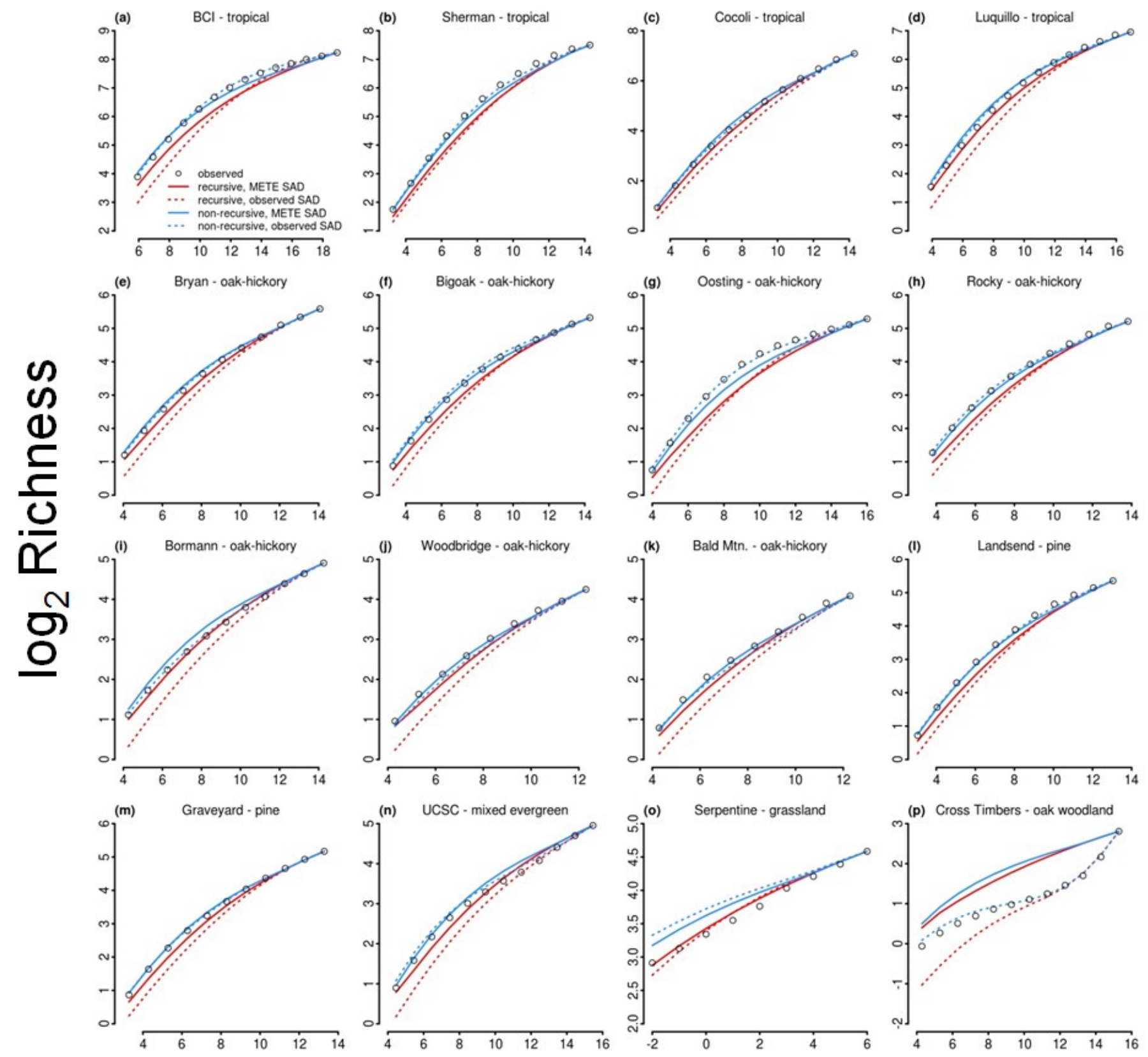

$\log _{2}$ Area $\left(\mathrm{m}^{2}\right)$ 


\section{Figure 3}

Observed-predicted plots for species richness

Observed vs predicted richness across datasets and spatial scales for the four METE SAR models. The $R^{2}$-value is computed with respect to the one-to-one line (diagonal).
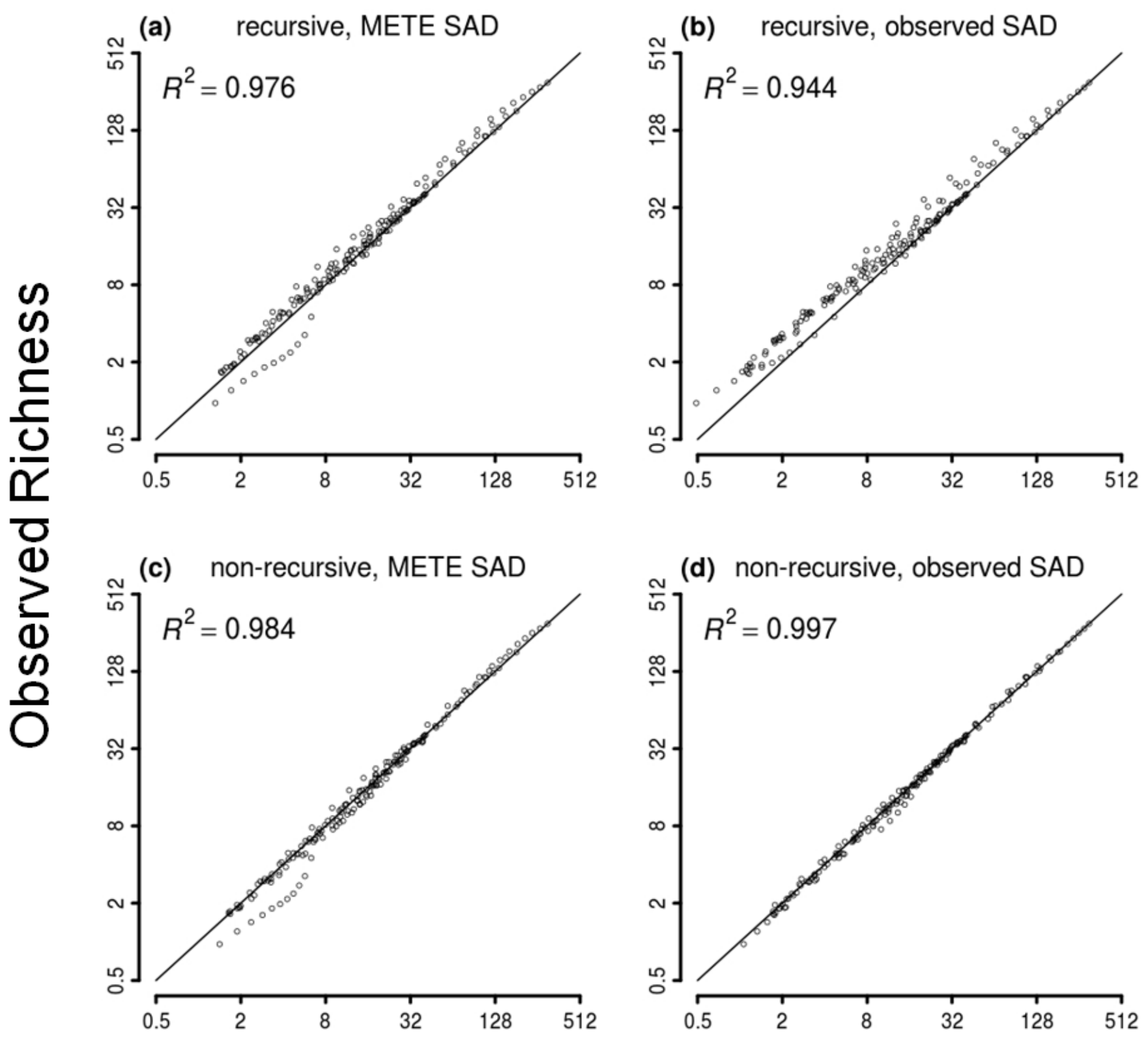

Predicted Richness 